\title{
The Hardy-Littlewood Maximal Type Operators between Banach Function Spaces
}

\author{
MieczysłaW Mastyło \& Carlos PÉrez
}

\begin{abstract}
We investigate variants of the maximal operator and show their applications to study boundedness of the classical HardyLittlewood maximal operator between weighted Banach function spaces which satisfy certain geometrical lattice conditions. We prove inequalities for rearrangement of the maximal operators generated by rearrangement invariant spaces. Applying this to the Lorentz spaces, we give new sufficient conditions for the boundedness of the Hardy-Littlewood maximal operator between weighted $L^{p}$-spaces with different weights. We also prove that under some mild hypotheses these conditions are also necessary.
\end{abstract}

\section{INTRODUCTION}

Maximal functions have proved to be tools of great importance in analysis and in particular in harmonic analysis. The main example is the Hardy-Littlewood maximal function, which is defined for locally integrable functions $f \in L_{\text {loc }}^{1}\left(\mathbb{R}^{n}\right)$ by

$$
M f(x)=\sup _{Q \ni x} \frac{1}{|Q|} \int_{Q}|f(y)| \mathrm{d} y, \quad x \in \mathbb{R}^{n},
$$

where the supremum is taken over all the cubes $Q$ containing $x$. Their study not only contains intrinsic interest but also intertwines with the study of singular integral operators, very particularly in the context of weighted norm inequalities (see $[7,8,10])$. As an example of the central role played by $M$, we mention the celebrated extrapolation theorem of Rubio de Francia. Indeed, the proof of this theorem is based on appropriate boundedness properties of the Hardy-Littlewood maximal function on weighted $L^{p}$-spaces. We refer to [5] for an overview of 
the theory and to [9] for a recent improvement of the extrapolation theorem. Therefore the study of weighted estimates with one or two weights for maximal functions will yield a better understanding for the corresponding situation for singular integral operators.

The starting point of the modern theory of weighted norm inequalities goes back to the paper of Muckenhoupt [15]. In that paper the inequality

$$
\int_{\mathbb{R}^{n}}(w(x) M f(x))^{p} \mathrm{~d} x \leq c \int_{\mathbb{R}^{n}}(v(x)|f(x)|)^{p} \mathrm{~d} x
$$

with $w=v$ was characterized in terms of the surprisingly simple geometric condition

$$
\sup _{Q}\left(\frac{1}{|Q|} \int_{Q} w(y)^{p} \mathrm{~d} y\right)^{1 / p}\left(\frac{1}{|Q|} \int_{Q} w(y)^{-p^{\prime}} \mathrm{d} y\right)^{1 / p^{\prime}}<\infty
$$

usually denoted by $A_{p}$, although the normalization we will be using in this paper on the weights differs from the usual one in harmonic analysis.

The study of the case when the weights are different is of interest for many reasons, and there is a revival of the area mainly because of its relationship with the theory of singular integrals. Sawyer obtained in [22] a characterization by means of the following $S_{p}$ condition: there is a positive constant $c$ such that for all cubes $Q$ we have

$$
\int_{Q}\left(M\left(v^{-p^{\prime}} \chi_{Q}\right)(y) w(y)\right)^{p} \mathrm{~d} y \leq c \int_{Q} v^{-p^{\prime}}(y) \mathrm{d} y .
$$

We note that in general Sawyer's $S_{p}$ condition is not so easy to verify in practice, especially if we compare it with the two weight $A_{p}$ condition (1.2) which is necessary and sufficient for the weak type boundedness, namely,

$$
\sup _{\lambda>0} \lambda^{p} w^{p}\left(\left\{x \in \mathbb{R}^{n}: M f(x)>\lambda\right\}\right) \leq C \int_{\mathbb{R}^{n}}(|f(x)| v(x))^{p} \mathrm{~d} x,
$$

as it is well known [7]. However, simple examples show (cf. [7, p. 395]) that this condition is not sufficient for the two weight problem (1.1), namely, we have the strict inclusion $S_{p} \subset A_{p}$. It is therefore natural to try to understand the "gap" between the two conditions. In particular, it would be interesting to obtain sufficient conditions "close" in form to $A_{p}$ due to its simplicity. The first author who studied this problem was Neugebauer in [16], where he proved that if $(w, v)$ is a couple of weights such that for some $r>1$

$$
\sup _{Q}\left(\frac{1}{|Q|} \int_{Q} w(y)^{p r} \mathrm{~d} y\right)^{1 / p r}\left(\frac{1}{|Q|} \int_{Q} v(y)^{-p^{\prime} r} \mathrm{~d} y\right)^{1 / p^{\prime} r}<\infty,
$$


where the supremum is taken over all cubes $Q$, then (1.1) holds. This condition is often called the "power bump" condition. The method in [16] is interesting because it shows that (1.4) is equivalent to "inserting" an $A_{p}$ weight between the given weights $w$ and $v$. However, it is not sharp enough to obtain better conditions.

The two weight problem was further considered in [19] by the second author, who used a different approach that allowed the generation of larger classes of couples of weights $(w, v)$ for which (1.1) holds. These larger classes contain Neugebauer's condition (1.4) as a particular case. The key idea is to replace the average norm associated to the weight $v^{-1}$ in (1.2) by an "stronger" norm defined in terms of any appropriate Banach function space whose associated space satisfies an appropriate $L^{p}$ boundedness. The main non-power bump example is given by

$$
\sup _{Q}\left(\frac{1}{|Q|} \int_{Q} w(y)^{p} \mathrm{~d} y\right)^{1 / p}\left\|v^{-1}\right\|_{X, Q}<\infty,
$$

where $X=L_{B}$ is the Orlicz space defined by the Young function $B$ and the average is given by

$$
\|f\|_{X, Q}=\|f\|_{B, Q}=\inf \left\{\lambda>0: \frac{1}{|Q|} \int_{Q} B\left(\frac{|f(y)|}{\lambda}\right) \mathrm{d} y \leq 1\right\} .
$$

The key point is to assume that the maximal operator corresponding to its associate space $X^{\prime}$, namely, $M_{X^{\prime}}$, is bounded on $L^{p}\left(\mathbb{R}^{n}\right)$. In the case that $X=L_{B}$ this is given by the tail condition:

$$
\int_{c}^{\infty}\left(\frac{t^{p^{\prime}}}{B(t)}\right)^{p-1} \frac{\mathrm{d} t}{t}<\infty,
$$

and no better than that. Interesting examples are given by $B(t) \approx t^{p^{\prime}}(\log t)^{p^{\prime}-1+\delta}$, or $B(t) \approx t^{p^{\prime}}(\log t)^{p^{\prime}-1}(\log (\log t))^{p^{\prime}-1+\delta}$, where $\delta>0$. Applications of these ideas can be found in $[4,6]$. We refer the reader to [5] for more information about classes of weights and for some other related results.

Besides its intrinsic interest, conditions like (1.5) carry some deep information because they are intimately related to some sharp endpoint estimate for singular integral operators. Indeed, it is shown in [18] that as a consequence of a special choice of $B$ in (1.7), for a given $\varepsilon>0$, there is $c>0$ depending on $\varepsilon$, the dimension and the smoothness of the kernel of $T$ such that

$$
\|T f\|_{L^{1, \infty}(w)} \leq \mathcal{C}_{\varepsilon, n, T} \int_{\mathbb{R}^{n}}|f(x)| M_{L(\log L)^{\varepsilon}}(w)(x) \mathrm{d} x, \quad w \geq 0 .
$$

It was conjectured in that paper that this result would be false in the case $\varepsilon=0$ where $M_{L(\log L)^{\varepsilon}}$ is replaced by $M$, but only recently this result was shown to be false by Reguera and Thiele in [21] (see also the previous work by Reguera in [20]). 
The purpose of this paper is to study the two weight problem for $M$ within the context of Banach function spaces. We believe that some of our results may lead to a better understanding of more difficult operators like singular integrals.

The paper is organized as follows. In Section 2, we introduce some fundamental notation. In Section 3, we prove the main result on the boundedness of the Hardy-Littlewood maximal operator between Banach function spaces. We involve a general variant of the Hardy-Littlewood maximal $M_{F}$ operator introduced in [19]. The operator $M_{F}$ is generated by a rearrangement invariant space on $\mathbb{R}^{n}$. We study the boundedness of this type of operator between Banach function spaces. This problem is motivated by the two weight problem for $M$ and by (1.8), and seems interesting on its own. In Section 3, we also prove estimates of the rearrangement the Hardy-Littlewood type maximal function generated by Lorentz space. These estimates allow us to provide a sufficient condition on rearrangement invariant spaces $F$ and $X$ on $\mathbb{R}^{n}$ which ensures that the maximal operator $M_{F}$ is bounded on $X$. As a consequence we derive that if $F$ is a rearrangement invariant space with fundamental function $\varphi_{F}$, then $M_{F}$ is bounded on $L^{p}\left(\mathbb{R}^{n}\right)$, $1<p<\infty$, provided

$$
\int_{0}^{1} s^{-1 / p} \mathrm{~d} \varphi_{F}(s)<\infty .
$$

Furthermore, we prove that if $\varphi_{F}$ is a submultiplicative function near zero, then the condition above is also necessary for boundedness of $M_{F}$ on $L^{p}\left(\mathbb{R}^{n}\right)$. As an application of our results, we are able to localize a class of couples of weights $(w, v)$ for which $M$ is bounded from $L^{p}\left(v^{p}\right)$ to $L^{p}\left(w^{p}\right)$. In particular, in the case of classical Lorentz space $L^{s, 1}$ with $1<s<\infty$, we recover the result proved in [19] in a different way.

\section{Notation ANd PRELIMINARIES}

In this paper we shall use the standard notation and terminology in the theory of Banach lattices. A Banach function space on a measure space $(S, \mu)$ is defined to be a Banach space $X$ which is a subspace of $L^{0}=L(\mu)$ (the topological linear space of all equivalence classes of the real Lebesgue measurable functions equipped with the topology of convergence in measure) such that there exists $u \in X$ with $u>0$ almost everywhere and if $|f| \leq|g|$ almost everywhere, where $g \in X$ and $f \in L^{0}$, then $f \in X$ and $\|f\|_{X} \leq\|g\|_{X}$.

Let $X$ be a Banach lattice on $(S, \mu)$ and $w \in L^{0}(\mu)$ be a weight, i.e., with $w>0$ almost everywhere on $S$. We define the weighted Banach function space $X(w)$ by $\|x\|_{X(w)}=\|x w\|_{X}$. The Banach function space $X$ is said to be order continuous if for every $x \in X$ and every sequence $\left(x_{n}\right)$ such that $0 \leq x_{n} \leq|x|$ and $x_{n} \downarrow 0$ almost everywhere, it holds that $\left\|x_{n}\right\|_{X} \rightarrow 0$. We say that $X$ satisfies the Fatou property whenever for any $x_{n} \in X$ and $x \in L^{0}$ such that $x_{n} \rightarrow x$ almost everywhere and $\sup _{n}\left\|x_{n}\right\|_{X}<\infty$, we have that $x \in X$ and $\|x\|_{X} \leq$ $\liminf { }_{n}\left\|x_{n}\right\|_{X}$. 
The Köthe dual space $X^{\prime}$ of $X$ is a collection of all elements $y \in L^{0}$ such that

$$
\|y\|_{X^{\prime}}=\sup \left\{\int_{S}|x y| \mathrm{d} \mu:\|x\|_{X} \leq 1\right\}<\infty .
$$

The space $X^{\prime}$ equipped with the norm $\|\cdot\|_{X^{\prime}}$ is a Banach function space with the Fatou property. It is well known that $X=X^{\prime \prime}$ with equality of norms whenever $X$ satisfies the Fatou property.

Given $f \in L^{0}$, its distribution function is defined by

$$
\mu_{f}(\lambda)=\mu(\{x \in S:|f(x)|>\lambda\}),
$$

and its decreasing rearrangement is defined by

$$
f^{*}(t)=f_{\mu}^{*}(t):=\inf \left\{\lambda \geq 0: \mu_{f}(\lambda) \leq t\right\}
$$

for $t \geq 0$.

A Banach function space $\left(X,\|\cdot\|_{X}\right)$ on $(S, \mu)$ is called admissible provided $\chi_{A} \in X$ for every measurable set $A$ of finite measure. In what follows, if $X$ is a Banach function space on $\mathbb{R}^{n}$ (i.e., on $\left(\mathbb{R}^{n}, \mu\right)$, where $\mu$ is the Lebesgue measure), we write $\varphi_{X}(|A|):=\left\|\chi_{A}\right\|$, where $\chi_{A}$ denotes the characteristic function of a measurable set $A$ and $|A|:=\mu(A)$. Here $\varphi_{X}$ is said to satisfy the $\Delta_{2}$-condition provided there exists a constant $C>0$ such that $\varphi_{X}(|2 Q|) \leq C \varphi_{X}(|Q|)$ for all cubes $Q$ with finite Lebesgue measure.

Important classes of admissible Banach function spaces $X$ are rearrangement invariant (r.i.) spaces. Recall that $X$ is called an r.i. space provided $\mu_{f}=\mu_{g}$, $f \in X$ implies $g \in X,\|f\|_{X}=\|g\|_{X}$. Clearly $\left\|\chi_{A}\right\|_{X}$ depends only on $\mu(A)$. The function $\varphi_{X}(t):=\left\|\chi_{A}\right\|_{X}$, where $\mu(A)=t, 0 \leq t \leq \mu(S)$, is called the fundamental function of $X$.

Examples of r.i. Banach spaces are Marcinkiewicz and Lorentz spaces on $(S, \mu)$. Let $I:=[0, \mu(S))$ and $\varphi: I \rightarrow[0, \infty)$ be a quasi-concave function, that is, $\varphi(0)=0$ and $\varphi(t)>0$ for all $0<t \in I$ and both $\varphi$ and $t \mapsto \varphi_{*}(t):=t / \varphi(t)$ are non-decreasing functions on $I$. We note that a quasi-concave function $\varphi$ is equivalent to its least concave majorant $\tilde{\varphi}$; more exactly, $\frac{1}{2} \tilde{\varphi}(t) \leq \varphi(t) \leq \tilde{\varphi}(t)$ for all $t \in I$.

The Marcinkiewicz space $M(\varphi)$ is the r.i. space of all $f \in L^{0}(\mu)$ such that

$$
\|f\|_{M(\varphi)}:=\sup _{t \in I} \frac{1}{\varphi(t)} \int_{0}^{t} f^{*}(s) \mathrm{d} s<\infty .
$$

If $\varphi: I \rightarrow[0, \infty)$ is a non-decreasing concave function, with $\varphi(0)=0$, the Lorentz space $\Lambda(\varphi)$ consists of all $f \in L^{0}$ such that

$$
\|f\|_{\Lambda(\varphi)}=\int_{I} f^{*}(s) \mathrm{d} \varphi(s):=\varphi(0+) f^{*}(0+)+\int_{I} f^{*}(s) \varphi^{\prime}(s) \mathrm{d} s<\infty,
$$


where $\varphi^{\prime}$ denotes the derivative of $\varphi$ (which exists almost everywhere).

Notice here that the fundamental functions of these spaces are $\varphi_{\Lambda(\varphi)}=\varphi$ and $\varphi_{M(\varphi)}=\varphi_{*}$.

In the sequel we will need the well-known fact that if $X$ is an r.i. Banach space on $\mathbb{R}^{n}$ with the fundamental function $\varphi$, then $\varphi$ is quasi-concave and the following continuous inclusions hold (see [11, Theorems II.5.5 and II.5.7] or [3, Theorem II.5.13]):

$$
\Lambda(\tilde{\varphi}) \hookrightarrow X \hookrightarrow M\left(\varphi_{*}\right) .
$$

\section{MAin RESUlTS}

Let $X$ be a Banach function space on $\mathbb{R}^{n}$. Throughout the paper $X_{\text {loc }}$ denotes the space of all $f \in L^{0}$ such that $f X_{Q} \in X$ for every cube $Q$ in $\mathbb{R}^{n}$. Following [19], for every $f \in X_{\text {loc }}$, we define the $X$-average by

$$
\|f\|_{X, Q}=\left\|\tau_{\ell(Q)}\left(f X_{Q}\right)\right\|_{X} .
$$

Here $\tau_{\delta}$ with $\delta>0$ is the dilatation operator defined for all $f \in L^{0}$ by $\tau_{\delta} f(x)=$ $f(\delta x)$, and $\ell(Q)$ is the side-length of the cube $Q$. We define the maximal operator $M_{X}$ associated to the space $X$ by

$$
M_{X} f(x)=\sup _{Q \ni X}\|f\|_{X, Q}, \quad f \in X_{\mathrm{loc}},
$$

where the supremum is taken over all the cubes $Q$ containing $x$.

We observe that in the case of the Orlicz space $L_{\varphi}$ on $\mathbb{R}^{n}$, the maximal operator $M_{\varphi}:=M_{L_{\varphi}}$ is given by

$$
M_{\varphi} f(x)=\sup _{Q \ni X}\left\|f \chi_{Q}\right\|_{\varphi, Q},
$$

where

$$
\|f\|_{\varphi, Q}:=\inf \left\{\lambda>0: \frac{1}{|Q|} \int_{Q} \varphi\left(\frac{|f(x)|}{\lambda}\right) \mathrm{d} x \leq 1\right\} .
$$

In [1] it was proved that there exists a constant $c_{n}>0$ such that

$$
\left(M_{\varphi} f\right)^{*}(t) \leq c_{n}\left\|f^{*}\right\|_{\varphi,(0, t),} \quad t>0 .
$$

It is easy to see that the maximal operator $M_{p, q}:=M_{L^{p, q}}$ associated to the Lorentz $L^{p, q}$ on $\mathbb{R}^{n}$ with $1<p, q<\infty$ is given by

$$
M_{p, q} f(x)=\sup _{Q \ni x} \frac{1}{|Q|^{1 / p}}\left\|f \chi_{Q}\right\|_{p, q} .
$$


This operator was studied in $[2,12,23]$. It was proved in [2], using interpolation, that if $1 \leq q \leq p$, then there exists a constant $c>0$ such that

$$
\left(M_{p, q} f\right)^{*}(t) \leq \frac{c}{t^{1 / p}}\left(\int_{0}^{t} f^{*}(s)^{q} s^{q / p-1} \mathrm{~d} s\right)^{1 / q}, \quad t>0 .
$$

We extend the above-mentioned rearrangement estimates to the more general setting of maximal operators and show applications to study boundedness of the Hardy-Littlewood maximal operator.

For formulation of the first result on boundedness of the maximal operator, some additional notation and definitions are required. Given a Banach function space $X$ and $1<p<\infty$, we define the $p$-concavification $X_{p}$ to be a quasi-Banach function space of all $x \in L^{0}(\mu)$ such that $|x|^{1 / p} \in X$, equipped with the quasinorm $\|x\|_{X_{p}}=\left\||x|^{1 / p}\right\|_{X}^{p}$. The Banach function space $X$ is said to be $p$-convex if there exists a constant $C>0$ such that for any $x_{1}, \ldots, x_{n} \in X$, we have

$$
\left\|\left(\sum_{k=1}^{n}\left|x_{k}\right|^{p}\right)^{1 / p}\right\|_{X} \leq C\left(\sum_{k=1}^{n}\left\|x_{k}\right\|_{X}^{p}\right)^{1 / p} .
$$

The least $C$ is denoted by $M^{(p)}(X)$.

Note that if $X$ is $p$-convex, then for $x \in X_{p}$ and $x_{1}, \ldots, x_{n} \in X_{p}$ with $|x| \leq \sum_{k=1}^{n}\left|x_{k}\right|$, we have

$$
\begin{aligned}
\|x\|_{X_{p}} & \leq\left\|\left(\sum_{k=1}^{n}\left(\left|x_{k}\right|^{1 / p}\right)^{p}\right)^{1 / p}\right\|_{X_{p}} \leq M^{(p)}(X)^{p} \sum_{k=1}^{n}\left\|\left|x_{k}\right|^{1 / p}\right\|_{X}^{p} \\
& =M^{(p)}(X)^{p} \sum_{k=1}^{n}\left\|x_{k}\right\|_{X_{p}} .
\end{aligned}
$$

This shows that the lattice norm $\|\cdot\|^{*}$ defined on $X_{p}$ by

$$
\|x\|^{*}:=\inf \left\{\sum_{k=1}^{n}\left\|x_{k}\right\|_{X_{p}}:|x| \leq \sum_{k=1}^{n}\left|x_{k}\right|, n \in \mathbb{N}\right\}, \quad x \in X_{p},
$$

satisfies

$$
M^{(p)}(X)^{-p}\|\cdot\|_{X_{p}} \leq\|\cdot\|^{*} \leq\|\cdot\|_{X_{p}} .
$$

In particular, this implies a well-known fact: if a Banach function space $X$ on $(S, \mu)$ is $p$-convex $(1<p<\infty)$, then there exists a Banach function space $Y$ on $(S, \mu)$ such that $x \in X$ if and only if $|x|^{p} \in Y$ and

$$
M^{(p)}(X)^{-1}\|x\|_{X} \leq\left\||x|^{p}\right\|_{Y}^{1 / p} \leq\|x\|_{X} .
$$

This fact will be used below in the proof without any references. 
A Banach lattice $X$ is said to satisfy a lower $p$-estimate, $1<p<\infty$, if there exists a constant $C$ such that

$$
\left(\sum_{i=1}^{n}\left\|x_{i}\right\|_{E}^{p}\right)^{1 / p} \leq C\left\|\sum_{i=1}^{n} x_{i}\right\|_{E}
$$

for every finite set of pairwise disjoint elements $\left\{x_{1}, \ldots, x_{n}\right\}$ in $X$ (see [14, 1.f.4]). The least $C$ is denoted by $\ell_{(p)}(X)$. [19].

The following theorem is an extension of the main result, Theorem 1.2, from

Theorem 3.1. Let $E, X$, and $Y$ be Banach function spaces over $\mathbb{R}^{n}$ where $E, X$ are admissible, and let $(w, v)$ be a couple of weights on $\mathbb{R}^{n}$. If the maximal operator $M_{E^{\prime}}$ associated to $E^{\prime}$ is bounded on $X, X$ satisfies a lower $p$-estimate, $Y$ is $p$-convex for some $1<p<\infty, \varphi_{X}$ satisfies the $\Delta_{2}$-condition, and

$$
\sup _{\mathcal{Q} \ni Q} \frac{\varphi_{Y(w)}(|Q|)}{\varphi_{X}(|Q|)}\left\|v^{-1}\right\|_{E, Q}<\infty
$$

then the Hardy-Littlewood maximal operator $M$ is bounded from $X(v)$ into $Y(w)$.

Proof. Our hypothesis that $X(w)$ satisfies a lower $p$-estimate implies that $X(w)$ is order continuous and so a separable space. Thus the set of bounded functions with compact support is dense in $X(w)$. Fix $f \in X(w)_{b}$ and $a>2^{n}$. For each integer $k$, we set

$$
\begin{aligned}
& \Omega_{k}=\left\{x \in \mathbb{R}^{n}: a^{k}<M f(x)\right\}, \\
& D_{k}=\left\{x \in \mathbb{R}^{n}: M^{d} f(x)>\frac{a^{k}}{4^{n}}\right\} .
\end{aligned}
$$

Applying the classical Calderón-Zygmund decomposition [7, p. 137], we conclude that there is a family of maximal non-overlapping dyadic cubes $\left\{Q_{k, j}\right\}$ such that $\Omega_{k} \subset \bigcup_{j} 3 Q_{k, j}, D_{k}=\bigcup_{j} Q_{k, j}$, and

$$
\frac{a^{k}}{4^{n}}<\frac{1}{\left|Q_{k, j}\right|} \int_{Q_{k, j}} f(y) \mathrm{d} y \leq \frac{a^{k}}{2^{n}} .
$$

Without loss of generality, we may assume that the $M^{(p)}(Y)=1$ and so there exists a Banach function space $\tilde{Y}$ on $\mathbb{R}^{n}$ such that $y \in Y$ if and only if $|x|^{p} \in \tilde{Y}$ and $\|x\|_{Y}=\left\||x|^{p}\right\|_{\tilde{Y}}^{1 / p}$. Combining the above yields that for some universal 
constants $C_{j}(j=1, \ldots, 4)$, we have

$$
\begin{aligned}
\|M f\|_{Y(w)} & =\left\|((M f) w)^{p}\right\|_{\bar{Y}}^{1 / p}=\left\|\sum_{k}((M f) w)^{p} \chi_{\Omega_{k} \backslash \Omega_{k+1}}\right\|_{\tilde{Y}}^{1 / p} \\
& \leq C_{1}\left\|\sum_{k} a^{k p} w^{p} \chi_{\Omega_{k} \backslash \Omega_{k+1}}\right\|_{\tilde{Y}}^{1 / p} \\
& \leq C_{2}\left\|\sum_{k, j}\left(\frac{1}{\left|Q_{k, j}\right|} \int_{Q_{k, j}}|f(y)| \mathrm{d} y\right)^{p} w^{p} \chi_{3 Q_{k, j}}\right\|_{\tilde{Y}}^{1 / p} \\
& \leq C_{3}\left\|\sum_{k, j}\left(\frac{1}{\left|3 Q_{k, j}\right|} \int_{3 Q_{k, j}}|f(y)| v(y) v(y)^{-1} \mathrm{~d} y\right)^{p} w^{p} \chi_{3 Q_{k, j}}\right\|_{\tilde{Y}}^{1 / p} \\
& \leq C_{4}\left(\sum_{k, j}\left(\frac{1}{\left|3 Q_{k, j}\right|} \int_{3 Q_{k, j}}|f(y)| v(y) v(y)^{-1} \mathrm{~d} y\right)^{p}\left\|\chi_{3 Q_{k, j}}\right\|_{Y(w)}^{p}\right)^{1 / p} .
\end{aligned}
$$

For any integers $k, j$, we put $E_{k, j}=Q_{k, j} \backslash Q_{k, j} \cap D_{k+1}$, and by [19, Lemma 4.2] there exists a constant $\beta>0$ such that $\left|Q_{k, j}\right|<\beta\left|E_{k, j}\right|$ for each $k, j$. The $\Delta_{2}$-condition for $\varphi_{X}$ gives that there exists a constant $C_{\beta}>0$ such that for all $k, j$

$$
\varphi_{X}\left(\left|3 Q_{k, j}\right|\right) \leq C_{\beta} \varphi_{X}\left(\left|E_{k, j}\right|\right)=C_{\beta}\left\|\chi_{E_{k, j}}\right\|_{X}
$$

For all cubes $Q, g \in E_{\mathrm{loc}}$ and $h \in E_{\mathrm{loc}}^{\prime}$, we have

$$
\frac{1}{|Q|} \int_{Q} g(y) h(y) \mathrm{d} y \leq\|g\|_{X, Q}\|h\|_{X^{\prime}, Q} .
$$

Thus the combination of the inequalities shown above with our hypotheses on $M_{E^{\prime}}$ and $X$ implies that the following estimates hold with some universal constants $\tilde{C}_{j}$, $j=1,2,3$ :

$$
\begin{aligned}
& \|M f\|_{Y(w)} \leq \tilde{C}_{1}\left(\sum_{k, j}\|f v\|_{E^{\prime}, 3 Q_{k, j}}^{p}\left\|v^{-1}\right\|_{E, 3 Q_{k, j}}^{p}\left\|X_{3 Q_{k, j}}\right\|_{Y(w)}^{p}\right)^{1 / p} \\
& \quad \leq \tilde{C}_{2}\left(\sum_{k, j}\|f v\|_{E^{\prime}, 3 Q_{k, j}}^{p}\left\|v^{-1}\right\|_{E, 3 Q_{k, j}}^{p}\left(\frac{\varphi_{Y(w)}\left(\left|3 Q_{k, j}\right|\right)}{\varphi_{X}\left(\left|3 Q_{k, j}\right|\right)}\right)^{p} \varphi_{X}\left(\left|3 Q_{k, j}\right|\right)^{p}\right)^{1 / p} \\
& \quad \leq \tilde{C}_{2} C_{\beta}\left(\sum_{k, j}\left\|M_{E^{\prime}}(f v) \chi_{E_{k, j}}\right\|_{X}^{p}\right)^{1 / p} \\
& \leq \tilde{C}_{2} C_{\beta} \ell_{(p)}(X)\left\|\sup _{k, j} M_{E^{\prime}}(f v) \chi_{E_{k, j}}\right\|_{X} \\
& \leq \tilde{C}_{3}\left\|M_{E^{\prime}}(f v)\right\|_{X} \leq \tilde{C}_{3}\|f\|_{X(v) .}
\end{aligned}
$$


We conclude by discussing some applications. We will need some rearrangement inequalities for some nonstandard maximal operators investigated in the paper [13]. We recall some notation introduced in this paper. If $F$ is a non-negative set function defined on the collection $\mathcal{B}$ of all sets of positive finite Lebesgue measure, then its maximal function is given by

$$
M F(x)=\sup _{Q \ni x} F(Q)
$$

where the supremum is taken over all cubes $Q \in \mathcal{Q}$ containing $x$, where $\mathcal{Q}$ denotes the set of all cubes $Q$ such that $0<|Q|<\infty$.

A set function $F$ is said to be pseudo-increasing with a constant $c$ if there exists a constant $c>0$ such that for any finite collection of pairwise disjoint cubes $\left\{Q_{i}\right\}$, we have

$$
\min _{i} F\left(Q_{i}\right) \leq c F\left(\bigcup_{i} Q_{i}\right) .
$$

If $c=1$, then $F$ is said to be increasing.

An interesting result obtained by Lerner in [13] states that if $F$ is a pseudoincreasing set function with constant $c$, then for any $t>0$

$$
(M F)^{*}(t) \leq c \sup _{|A|>t / 3^{n}} F(A),
$$

where the supremum is taken over all sets $A$ of finite Lebesgue measure $|A|>$ $t / 3^{n}$.

Throughout the rest of the paper, an r.i. space on $\mathbb{R}^{n}$ is said to be generated by an r.i. space $\tilde{X}$ on $(0, \infty)$ provided $f \in X$ if and only if $f^{*} \in \tilde{X}$ and $\|f\|_{X}=$ $\left\|f^{*}\right\|_{\bar{X}}$. It is well known that any r.i. space $X$ on $\mathbb{R}^{n}$ with the Fatou property is generated by some r.i. space on $[0, \infty)$ (see [3]).

For every $0<t<\infty$, a dilatation operator $D_{t}$ is defined for a Lebesgue measurable function $f$ on $(0, \infty)$ by

$$
D_{s} f(t)=f(t / s), \quad 0<t<\infty .
$$

It is a well-known fact that $D_{s}$ is bounded on every r.i. space on $(0, \infty)($ see $[3,11$, 14]). Thus, by $D_{r s}=D_{r} D_{s}$, we have $\left\|D_{r s}\right\| \leq\left\|D_{r}\right\|\left\|D_{s}\right\|$ for all $r, s>0$.

A consequence of the mentioned Lerner result is the following corollary on the rearrangement inequality of the maximal operator $M_{X}$.

Proposition 3.2. Let $X$ be an r.i. space on $\mathbb{R}^{n}$ generated by the r.i. space $\tilde{X}$ on $(0, \infty)$. Assume the set function $\mathcal{B} \ni A \mapsto\left\|\tau_{|A|^{1 / n}}\left(f X_{A}\right)\right\|_{X}$ generated by a fixed $f \in X_{\text {loc }}$ is pseudo-increasing with a constant $c$. Then, for every $t>0$, we have

$$
\left(M_{X} f\right)^{*}(t) \leq c\left\|\left(D_{3^{n} / t} f^{*}\right) \chi_{(0,1)}\right\|_{\bar{X}} .
$$


Proof. Let $F(A):=\left\|\boldsymbol{T}_{|A|^{1 / n}}\left(f \chi_{A}\right)\right\|_{X}$ for all $A \in \mathcal{B}$. It follows by the Lerner result that for any $t>0$, we have

$$
(M F)^{*}(t) \leq c \sup \left\{F(A): A \in \mathcal{B},|A|>\frac{t}{3^{n}}\right\} .
$$

For all $s>0, A \in \mathcal{B}$, we have $\tau_{|A|^{1 / n}}\left(f \chi_{A}\right)^{*}(s)=\left(f \chi_{A}\right)^{*}(s|A|),\left(f \chi_{A}\right)^{*} \leq$ $f^{*} \chi_{(0,|A|)}$. This implies

$$
\left.F(A)=\| D_{1 /|A|}\left(f \chi_{A}\right)^{*}\right)\left\|_{\tilde{X}} \leq\right\| D_{1 /|A|}\left(f^{*} \chi_{(0,|A|)}\right)\left\|_{\bar{X}}=\right\|\left(D_{1 /|A|} f^{*}\right) \chi_{(0,1)} \|_{\tilde{X}} .
$$

Combining the above inequalities, we obtain

$$
(M F)^{*}(t) \leq c\left\|\left(D_{3^{n} / t} f^{*}\right) \chi_{(0,1)}\right\|_{\bar{X}} .
$$

The obvious equality $M_{X} f=M F$ completes the proof.

We will present new examples of pseudo-increasing set functions and show applications to the Hardy-Littlewood maximal type operators. We recall the definition of the generalized Orlicz space. Let $E$ be a Banach lattice on $(S, \mu)$, and let $\varphi:[0, \infty) \rightarrow[0, \infty)$ be an Orlicz function, i.e., $\varphi$ is convex with $\varphi(t)=0$ if and only if $t=0$. The generalized Orlicz space

$$
X_{\varphi}:=\left\{x \in L^{0}(\mu): \varphi\left(\frac{|x|}{\lambda}\right) \in X \text { for some } \lambda>0\right\}
$$

is a Banach function space under the norm

$$
\|x\|_{X_{\varphi}}:=\inf \left\{\lambda>0:\left\|\varphi\left(\frac{|x|}{\lambda}\right)\right\|_{X} \leq 1\right\} .
$$

If $X=\Lambda(\psi)$ is the Lorentz space, then $\Lambda(\psi)_{\varphi}$ is called the Orlicz-Lorentz space and is denoted by $\Lambda_{\varphi, \psi}$. In particular, $\left(L_{1}\right)_{\varphi}$ is the classical Orlicz space equipped with the Luxemburg norm and is denoted by $L_{\varphi}$.

Lemma 3.3. Let $X_{\varphi}$ be a generalized Orlicz space on $\mathbb{R}^{n}$ and $\rho:(0, \infty) \rightarrow$ $(0, \infty)$ be a function. Assume that for every $f \in X_{\text {loc }}$ the set function $\mathcal{B} \ni A \mapsto$ $\left\|\tau_{\rho(|A|)}\left(f X_{A}\right)\right\|_{X}$ is pseudo-increasing with a constant $c$. Then the set function

$$
\mathcal{B} \ni A \mapsto\left\|\tau_{\rho(|A|)}\left(f X_{A}\right)\right\|_{X_{\varphi}}
$$

is also pseudo-increasing with a constant $c_{1}=\min \{1, c\}$.

Proof. For $f \in\left(X_{\varphi}\right)_{\text {loc }}$ with $f \neq 0$, we define the set function $F: \mathcal{B} \rightarrow[0, \infty)$ by

$$
F(A):=\left\|\tau_{\rho(|A|)}\left(f \chi_{A}\right)\right\|_{X_{\varphi}}, \quad A \in \mathcal{B} .
$$


Let $\left\{Q_{i}\right\}$ be a finite collection of pairwise disjoint cubes. Without loss of generality we may assume that $0<\min _{i} F\left(Q_{i}\right)$. Fix $0<\varepsilon<\min _{i} F\left(Q_{i}\right)$, and set $\lambda=$ $\min _{i} F\left(Q_{i}\right)-\varepsilon$. Using the convexity of $\varphi$ and our hypothesis yields

$$
\begin{aligned}
\left\|\varphi\left(\tau_{\rho\left(\left|\cup_{i} Q_{i}\right|\right)}\left(\frac{f X_{\cup_{i} Q_{i}}}{c_{1} \lambda}\right)\right)\right\|_{X} & =\left\|\tau_{\rho\left(\left|\cup_{i} Q_{i}\right|\right)}\left(\varphi\left(\frac{|f|}{c_{1} \lambda}\right) \chi_{\cup_{i} Q_{i}}\right)\right\|_{X} \\
& \geq c \min _{i}\left\|\tau_{\rho\left(\left|Q_{i}\right|\right)}\left(\varphi\left(\frac{\left|f \chi_{Q_{i}}\right|}{c_{1} \lambda}\right)\right)\right\|_{X} \\
& \geq \frac{c}{c_{1}} \min _{i}\left\|\tau_{\rho\left(\left|Q_{i}\right|\right)}\left(\varphi\left(\frac{\left|f X_{Q_{i}}\right|}{F\left(Q_{i}\right)}-\varepsilon\right)\right)\right\|_{X} \geq 1 .
\end{aligned}
$$

This implies that

$$
F\left(\bigcup_{i} Q_{i}\right) \geq c_{1} \lambda=c_{1}\left(\min _{i} F\left(Q_{i}\right)-\varepsilon\right)
$$

and so by letting $\varepsilon \rightarrow 0$, we obtain the required inequality.

Lemma 3.4. Let $E:=\Lambda_{\varphi, \psi}$ be the Orlicz-Lorentz space on $\mathbb{R}^{n}$ generated by Orlicz function $\varphi$ and concave function $\psi$ with $\psi(0+)=0$. Then, for every $t>0$, the maximal operator $M_{E}$ associated to $E$ satisfies the rearrangement inequality

$$
\left(M_{E} f\right)^{*}(t) \leq\left\|\left(D_{3^{n} / t} f^{*}\right) \chi_{(0,1)}\right\|_{\tilde{E}}, \quad f \in E_{\mathrm{loc}},
$$

where $\tilde{E}$ is the Orlicz-Lorentz space $\Lambda_{\varphi, \psi}$ on $(0, \infty)$. In particular, for any $f \in$ $\Lambda(\psi)_{\mathrm{loc}}$ and $t>0$, we have

$$
\left(M_{\Lambda(\psi)} f\right)^{*}(t) \leq \int_{0}^{1} f^{*}\left(\frac{s t}{3^{n}}\right) \mathrm{d} \psi(s) .
$$

Proof. Note that the following formula holds (see [11, formula (5.4)]):

$$
\|f\|_{\Lambda(\psi)}=\int_{0}^{\infty} \psi\left(\mu_{f}(s)\right) \mathrm{d} s, \quad f \in \Lambda(\psi) .
$$

Given $f \in \Lambda(\psi)_{\text {loc }}$, we define the set function $F: \mathcal{B} \rightarrow[0, \infty)$ by

$$
F(A)=\left\|\boldsymbol{\tau}_{|A|^{1 / n}}\left(f \chi_{A}\right)\right\|_{\Lambda(\psi)}, \quad A \in \mathcal{B} .
$$

Formula (3.1) implies

$$
F(A)=\int_{0}^{\infty} \psi\left(\frac{\mu_{f X_{A}}(s)}{|A|}\right) \mathrm{d} s, \quad A \in \mathcal{B} .
$$


We claim that the set function $F$ is increasing. In fact, for any pairwise disjoint sets $A$ and $B$ with finite Lebesgue measures, we have (by concavity of $\psi$ )

$$
\begin{aligned}
F(A \cup B) & =\int_{0}^{\infty} \psi\left(\frac{\left.\mu_{f_{X_{A \cup B}}(s)}^{|A \cup B|}\right) \mathrm{d} s}{}\right. \\
& =\int_{0}^{\infty} \psi\left(\frac{|A|}{|A|+|B|} \frac{\mu_{f \chi_{A}}(s)}{|A|}+\frac{|B|}{|A|+|B|} \frac{\mu_{f \chi_{B}}(s)}{|B|}\right) \mathrm{d} s \\
& \geq \frac{|A|}{|A|+|B|} F(A)+\frac{|B|}{|A|+|B|} F(B) \geq \min \{F(A), F(B)\} .
\end{aligned}
$$

This concludes the proof, by Lemma 3.3 and Proposition 3.2.

We will need the following lemma (see [11, p. 136]).

Lemma 3.5. If $E$ is an r.i. space on $(0, \infty)$ and $\psi$ is a nondecreasing continuous function on $(a, b), 0 \leq a<b \leq \infty$, then, for all $x \in E$, we have

$$
\left\|\int_{a}^{b} D_{1 / s} x^{*}(t) \mathrm{d} \psi(s)\right\|_{E} \leq \int_{a}^{b}\left\|D_{1 / s} x\right\|_{E} \mathrm{~d} \psi(s) .
$$

Now we are ready to present the sufficient conditions for boundedness of the maximal operator $M_{E}$ on r.i. spaces.

Theorem 3.6. Assume that r.i. spaces $F$ and $X$ on $\mathbb{R}^{n}$ are such that $\varphi_{F}(0+)=0$, $X$ is generated by an r.i. space $\tilde{X}$, and

$$
\int_{0}^{1}\left\|D_{1 / s}\right\|_{\tilde{X} \rightarrow \bar{X}} \mathrm{~d} \varphi_{F}(s)<\infty .
$$

Then the following statements hold true for the maximal operator $M_{F}$ associated to $F$ :

(i) If $\varphi_{X}(0+)=0$, then there exists a constant $C>0$ such that

$$
\left\|M_{F} f\right\|_{X} \leq C\|f\|_{X}, \quad f \in L^{1} \cap L^{\infty} .
$$

(ii) If $X$ is separable, then the maximal operator $M_{F}$ is bounded on $X$.

Proof. (i) Since $\varphi_{F}$ is equivalent to its concave minorant, we may assume without loss of generality that $\varphi_{F}$ is a concave function. Then the inclusion map $\Lambda\left(\varphi_{F}\right) \hookrightarrow F$ has norm 1 , and so for all $x \in \mathbb{R}^{n}$ and $f \in L^{1} \cap L^{\infty}$,

$$
M_{F} f(x) \leq M_{\Lambda\left(\varphi_{F}\right)} f(x) .
$$

From Lemma 3.4 it follows that, for any $t>0$, we have

$$
\left(M_{F} f\right)^{*}(t) \leq \int_{0}^{1} f^{*}\left(\frac{s t}{3^{n}}\right) \mathrm{d} \varphi_{F}(s) .
$$


Combining this inequality with Lemma 3.5, we deduce that for $C:=\left\|D_{3^{n}}\right\|_{\tilde{X} \rightarrow \bar{X}}$ we obtain

$$
\begin{aligned}
\left\|M_{F} f\right\|_{X} & =\left\|\left(M_{F} f\right)^{*}\right\|_{\tilde{X}} \leq\left\|\int_{0}^{1} D_{1 / s}\left(D_{3^{n}} f^{*}\right) \mathrm{d} \varphi_{F}(s)\right\|_{\tilde{X}} \\
& \leq \int_{0}^{1}\left\|D_{1 / s}\left(D_{3^{n}} f^{*}\right)\right\|_{\tilde{X}} \mathrm{~d} \varphi_{F}(s) \\
& \leq C\left(\int_{0}^{1}\left\|D_{1 / s}\right\| \mathrm{d} \varphi_{F}(s)\right)\|f\|_{X},
\end{aligned}
$$

for all $f \in L^{1} \cap L^{\infty}$, as desired.

(ii) Note that $M_{F}$ is a positive sublinear operator, and so for all $f, g \in F_{\text {loc }}$, we have

$$
\left|M_{F} f-M_{F} g\right| \leq M_{F}(f-g), \quad \text { almost everywhere. }
$$

Since $X$ is separable, it follows that $\varphi_{X}(0+)=0$ and $L^{1} \cap L^{\infty}$ is a dense set in $X$. Thus the above estimate allows us to extend the inequality obtained in (i) for all $f \in X$ by density.

If $1<p<\infty$ and $X=L^{p}\left(\mathbb{R}^{n}\right)$, then $X$ is separable and $\tilde{X}=L^{p},\left\|D_{S}\right\|_{\tilde{X} \rightarrow \tilde{X}}=$ $s^{1 / p}$ for all $s>0$. Thus, applying Theorem 3.6, we obtain the following result.

Corollary 3.7. If $1<p<\infty$ and $F$ is an r.i. space on $\mathbb{R}^{n}$ such that

$$
\int_{0}^{1} s^{-1 / p} \mathrm{~d} \varphi_{F}(s)<\infty,
$$

then the maximal operator $M_{F}$ associated to $F$ is bounded on $L^{p}\left(\mathbb{R}^{n}\right)$.

We show that for a large class of r.i. spaces $F$, the condition shown above in Lemma 3.7 is also necessary for the boundedness of the maximal operator $M_{F}$ on $L^{p}\left(\mathbb{R}^{n}\right)$ with $1<p<\infty$. To do this it is convenient to use an index $\gamma_{F}$ defined for any r.i. space $F$ on $\mathbb{R}^{n}$ by

$$
\gamma_{F}=\limsup _{s \rightarrow 0+} \frac{\log s}{\log \varphi_{F}(s)} .
$$

Corollary 3.8. If $F$ is an ri. space on $\mathbb{R}^{n}$ with $\gamma_{F}<\infty$, then the maximal operator $M_{F}$ associated to $F$ is bounded on $L^{p}\left(\mathbb{R}^{n}\right)$ for every $\gamma_{F}<p<\infty$.

Proof. Let $q$ be a positive number with $\gamma_{F}<q<p<\infty$. Then it follows from the definition of $\gamma_{F}$ that there exist $C>0$ and $0<\delta<1$ such that

$$
\varphi_{F}(s) \leq C s^{1 / q}, \quad 0<s \leq \delta .
$$


Since $\varphi_{F}(0+)=0,\left(\varphi_{F}\right)^{\prime}(s) \leq \varphi_{F}(s) / s$ for almost all $s>0$. This implies

$$
\int_{0}^{\delta} s^{-1 / p} \mathrm{~d} \varphi_{F}(s) \leq \int_{0}^{\delta} s^{-1 / p-1} \varphi_{F}(s) \mathrm{d} s \leq \frac{C}{q} \int_{0}^{1} s^{1 / q-1 / p-1} \mathrm{~d} s<\infty
$$

and hence

$$
\int_{0}^{1} s^{-1 / p} \mathrm{~d} \varphi_{F}(s)<\infty .
$$

Thus Corollary 3.7 applies.

Theorem 3.9. Let $F$ be an r.i. space on $\mathbb{R}^{n}$ with $\gamma_{F}<\infty$. If $1<p<\infty$ and $\varphi_{F}$ is submultiplicative near zero (i.e., there exist $c>0$ and $s_{0}>0$ such that $\varphi_{F}(s t) \leq c \varphi_{F}(s) \varphi_{F}(t)$ for all $0<s \leq s_{0}$ and $\left.t>0\right)$, then the following conditions are equivalent:

(i) $M_{F}$ is bounded on $L^{p}\left(\mathbb{R}^{n}\right)$.

(ii) There exists $c>0$ such that $\int_{0}^{c}\left(\varphi_{F}(s)^{p} / s^{2}\right) \mathrm{d} s<\infty$.

(iii) $\gamma_{F}<p$.

(iv) $\int_{0}^{1} s^{-1 / p} \mathrm{~d} \varphi_{F}(s)<\infty$.

Proof. The implication (i) $\Longrightarrow$ (ii) follows from [19, Theorem 3.1].

(ii) $\Longrightarrow$ (iii) Put $\varphi(t):=c \varphi_{F}(t) / \varphi_{F}(1)$ for $t \geq 0$. Then our hypothesis on $\varphi_{F}$ implies $\varphi(1)=1$ and $\varphi(s t) \leq \varphi(s) \varphi(t)$ for all $0<s \leq s_{0}$ and $t>0$.

Let $\bar{\varphi}:[0, \infty) \rightarrow[0, \infty)$ be a submultiplicative function on $[0, \infty)$ defined by

$$
\bar{\varphi}(s)=\sup _{t>0} \frac{\varphi(s t)}{\varphi(t)}, \quad s \geq 0 .
$$

Then we get (see, e.g., [3, Chapter 5] or [11, Chapter 2]) that there exists $\alpha \geq 0$ such that

$$
\alpha=\sup _{0<s<1} \frac{\log \bar{\varphi}(s)}{\log s}=\lim _{s \rightarrow 0+} \frac{\log \bar{\varphi}(s)}{\log s} .
$$

Since $\bar{\varphi}(s)=\varphi(s)$ for all $0<s<s_{0}$, our hypothesis $\gamma_{F}<\infty$ implies $\alpha=1 / \gamma_{F}>$ 0 . Thus there exists $\delta>0$ such that

$$
s^{1 / \gamma_{F}} \leq \varphi(s), \quad 0<s<\delta,
$$

and hence we clearly have $\gamma_{F}<p$ provided that condition (ii) holds.

The implications (iii) $\Longrightarrow$ (iv) and (iv) $\Longrightarrow$ (i) follow by Corollary 3.8 and Corollary 3.7, respectively.

Analysis of the proofs of Corollary 3.8 and Theorem 3.9 gives the following result, which seems interesting on its own. 
Theorem 3.10. If $F$ is an ri. space on $\mathbb{R}^{n}$ with $\gamma_{F}<\infty$, then the following formulas hold:

$$
\begin{aligned}
\gamma_{F} & =\inf \left\{p>1: M_{F} \text { is bounded on } L^{p}\left(\mathbb{R}^{n}\right)\right\} \\
& =\inf \left\{p>1: \int_{0}^{1} \frac{\varphi_{F}(s)^{p}}{s^{2}} \mathrm{~d} s<\infty\right\} \\
& =\inf \left\{p>1: \int_{0}^{1} s^{-1 / p} \mathrm{~d} \varphi_{F}(s)<\infty\right\} .
\end{aligned}
$$

To state the next result, we need the Köthe duality formulas for Lorentz and Marcinkiewicz spaces (cf. $[3,11]$ ). For the sake of completeness we show these formulas below. If $(S, \mu)$ is an atomless measure space, then for any concave function $\psi:[0, \mu(S)) \rightarrow[0, \infty)$ with $\psi(0)=0$, the following duality formulas with equality of norms hold true:

$$
\Lambda(\psi)^{\prime}=M(\psi) \quad \text { and } \quad M(\psi)^{\prime}=\Lambda(\psi) .
$$

An immediate consequence of the above Corollary 3.7, Theorem 3.6, and the duality formula $M\left(\varphi_{*}\right)^{\prime}=\Lambda\left(\widetilde{\varphi_{*}}\right)$ is the following theorem.

Theorem 3.11. Let $1<p<\infty$, and let $\varphi:[0, \infty) \rightarrow[0, \infty)$ be a quasi-concave function such that $\int_{0}^{1} s^{-1 / p} \mathrm{~d} \varphi_{*}(s)<\infty$. Suppose that $(w, v)$ is a couple of weights on $\mathbb{R}^{n}$ such that

$$
\sup _{Q \in \mathcal{Q}}\left(\frac{1}{|Q|} \int_{Q} w(y)^{p} \mathrm{~d} y\right)^{1 / p}\left\|v^{-1}\right\|_{M\left(\varphi_{*}\right), Q}<\infty .
$$

Then the maximal Hardy-Littlewood operator $M$ is bounded from $L^{p}\left(v^{p}\right)$ to $L^{p}\left(w^{p}\right)$.

We conclude with the following remark: it follows from Theorem 3.11 that in the class of r.i. spaces $X$ on $\mathbb{R}^{n}$ with the same fundamental function $\varphi$ satisfying the condition $\int_{0}^{1} s^{-1 / p} \mathrm{~d} \varphi_{*}(s)<\infty$, the largest class of couples of weights $(w, v)$ on $\mathbb{R}^{n}$ which satisfy the condition

$$
\sup _{Q \in \mathcal{Q}}\left(\frac{1}{|Q|} \int_{Q} w(y)^{p} \mathrm{~d} y\right)^{1 / p}\left\|v^{-1}\right\|_{X, Q}<\infty
$$

is generated when $X$ is the largest r.i. space with the fundamental function $\varphi$, i.e., when $X$ is the Marcinkiewicz space $M\left(\varphi_{*}\right)$.

Acknowledgements. The first author was partially supported by the National Science Centre (NCN), Poland, grant no. 2011/01/B/ST1/06243. The second author was supported by the Spanish Ministry of Science and Innovation grant MTM2009-08934 and by the Junta de Andalucía, grant FQM-4745. We thank the referee for very careful reading of the paper. 


\section{REFERENCES}

[1] R.J. BAgBY and J.D. PARSONS, Orlicz spaces and rearranged maximal functions, Math. Nachr. 132 (1987), 15-27. http://dx.doi.org/10.1002/mana.19871320103. MR910041 (88i:46039)

[2] J. BAStero, M. Milman, and F.J. RuiZ, Rearrangement of Hardy-Littlewood maximal functions in Lorentz spaces, Proc. Amer. Math. Soc. 128 (2000), no. 1, 65-74. http://dx.doi.org/10.1090/S0002-9939-99-05128-X. MR1641637 (2000c:42020)

[3] C. Bennett and R. Sharpley, Interpolation of Operators, Pure and Applied Mathematics, vol. 129, Academic Press Inc., Boston, MA, 1988. MR928802 (89e:46001)

[4] D.V. Cruz-URibe, J.M. MARTElL, and C. PÉREZ, Sharp two-weight inequalities for singular integrals, with applications to the Hilbert transform and the Sarason conjecture, Adv. Math. 216 (2007), no. 2, 647-676. http://dx.doi.org/10.1016/j.aim.2007.05.022. MR2351373 (2008k:42029)

[5] Weights, Extrapolation and the Theory of Rubio de Francia, Operator Theory: Advances and Applications, vol. 215, Birkhäuser/Springer Basel AG, Basel, 2011. http://dx.doi.org/10.1007/978-3-0348-0072-3. MR2797562 (2012f:42001)

[6] Sharp weighted estimates for classical operators, Adv. Math. 229 (2012), no. 1, 408-441. http://dx.doi.org/10.1016/j.aim.2011.08.013. MR2854179 (2012k:42020)

[7] J. García-Cuerva and J.L. Rubio de Francia, Weighted Norm Inequalities and Related Topics, North-Holland Mathematics Studies, vol. 116, North-Holland Publishing Co., Amsterdam, 1985. Notas de Matemática [Mathematical Notes], 104. MR807149 (87d:42023)

[8] L. GrafaKos, Modern Fourier analysis, 2nd ed., Graduate Texts in Mathematics, vol. 250, Springer, New York, 2009. http://dx.doi.org/10.1007/978-0-387-09434-2. MR2463316 (2011d:42001)

[9] T. HYTÖNEN and C. PÉREZ, Sharp weighted bounds involving $A_{\infty}$, Journal of Analysis and Partial Differential Equations (2011), to appear.

[10] J.-L. Journé, Calderón-Zygmund Operators, Pseudodifferential Operators and the Cauchy Integral of Calderón, Lecture Notes in Mathematics, vol. 994, Springer-Verlag, Berlin, 1983. MR706075 (85i:42021)

[11] S.G. KREǏN, Yu.I. PETUNĪN, and E.M. SEMËNOV, Interpolation of linear operators, Translations of Mathematical Monographs, vol. 54, American Mathematical Society, Providence, R.I., 1982. Translated from the Russian by J. Szücs. MR649411 (84j:46103)

[12] M.A. LECKBAND and C.J. NEUGEBAUER, Weighted iterates and variants of the Hardy-Littlewood maximal operator, Trans. Amer. Math. Soc. 279 (1983), no. 1, 51-61. http://dx.doi.org/10.2307/1999370. MR704601 (85c:42021)

[13] A.K. LERNER, A new approach to rearrangements of maximal operators, Bull. London Math. Soc. 37 (2005), no. 5, 771-777. http://dx.doi.org/10.1112/S0024609305004698. MR2164840 (2006d:42032)

[14] J. LindenstraUSS and L. TZAFRIRI, Classical Banach Spaces. II: Function Spaces, Ergebnisse der Mathematik und ihrer Grenzgebiete [Results in Mathematics and Related Areas], vol. 97, Springer-Verlag, Berlin, 1979. MR540367 (81c:46001)

[15] B. MUCKENHOUPT, Weighted norm inequalities for the Hardy maximal function, Trans. Amer. Math. Soc. 165 (1972), 207-226. http://dx.doi.org/10.2307/1995882. MR0293384 (45\#2461)

[16] C.J. Neugebauer, Inserting Ap-weights, Proc. Amer. Math. Soc. 87 (1983), no. 4, 644-648. http://dx.doi.org/10.2307/2043351. MR687633 (84d:42026)

[17] C. PÉREZ, Two weighted inequalities for potential and fractional type maximal operators, Indiana Univ. Math. J. 43 (1994), no. 2, 663-683. http://dx.doi.org/10.1512/iumj.1994.43.43028. MR1291534 (95m:42028)

[18] _ Weighted norm inequalities for singular integral operators, J. London Math. Soc. (2) 49 (1994), no. 2, 296-308. http://dx.doi.org/10.1112/jlms/49.2.296. MR1260114 (94m:42037) 
[19] - On sufficient conditions for the boundedness of the Hardy-Littlewood maximal operator between weighted $L^{p}$-spaces with different weights, Proc. London Math. Soc. (3) 71 (1995), no. 1, 135-157. http://dx.doi.org/10.1112/plms/s3-71.1.135. MR1327936 (96k:42023)

[20] M.C. Reguera, On Muckenhoupt-Wheeden conjecture, Adv. Math. 227 (2011), no. 4, 14361450. http://dx.doi.org/10.1016/j.aim.2011.03.009. MR2799801 (2012d:42038)

[21] M.C. Reguera and Ch. Thiele, The Hilbert transform does not map $L^{1}(M w)$ to $L^{1, \infty}(w)$, Math. Res. Lett. 19 (2012), no. 1, 1-7. MR2923171

[22] E.T. SAWYER, A characterization of a two-weight norm inequality for maximal operators, Studia Math. 75 (1982), no. 1, 1-11. MR676801 (84i:42032)

[23] E.M. STEIN, Editor's note: the differentiability of functions in $\mathbb{R}^{n}$, Ann. of Math. (2) 113 (1981), no. 2, 383-385. MR607898 (84j:35077)

KEY WORDS AND PHRASES: maximal operators, rearrangement estimate.

2000 Mathematics SubJECt Classification: 42B20, 42B25, 46 E30.

Received: May 15, 2011; revised: December 9, 2011.

MieCZysŁaW MASTYŁO:

Faculty of Mathematics and Computer Science

Adam Mickiewicz University Poznań,

and Institute of Mathematics

Polish Academy of Sciences (Poznań branch)

Umultowska 87

61-614 Poznań, Poland

E-MAIL: mastylo@amu.edu.pl

CARlos PÉreZ:

Departamento de Aálisis Matemático

Facultad de Matemáticas, Universidad de Sevilla

41080 Sevilla, Spain

E-MAIL: carlosperez@us.es 